\title{
Special issue: cellular omics methods in plant reproduction research
}

\author{
Thomas Dresselhaus $^{1} \cdot$ Dolf Weijers ${ }^{2}$
}

Published online: 25 February 2019

c) Springer-Verlag GmbH Germany, part of Springer Nature 2019

The past decade has seen a revolution in the development of technologies to study whole genomes, transcriptomes, epigenomes, proteomes and metabolomes at continuously increasing resolution and sensitivity. As a consequence, developmental and reproductive processes can now often be described at near-cellular resolution involving the isolation and analysis of single or of a few cells at very defined stages. This gives unprecedented insight into how processes are regulated and vary between cells and stages. While some developmental processes are regulated at the scale of organs, tissues or larger cell population, some require precise control at the individual cellular scale. This is particularly the case during plant reproduction, where single cells are specified to adopt functions and identities to support the development of gametes, to aid fertilization, to establish nurturing tissues and to generate an embryo. Therefore, few fields in plant biology depend on cellular-scale methodology as much as the reproduction field does. In this special issue of Plant Reproduction, 11 papers describe the development of methodology toward collecting genome-wide omics data on reproductive cells.

In addition to becoming a reference for methods in the field of plant reproduction, this special issue also marks the introduction of a new category format in the journal Plant Reproduction aiming to provide the research community with the possibility of publishing up-to-date methodology. Method Papers will help to catalyze the widespread use of new and innovative technology and help to support newcomers in the field and to further advance the exciting fields in plant reproduction research.

Thomas Dresselhaus

thomas.dresselhaus@ur.de

Dolf Weijers

dolf.weijers@wur.nl

1 Cell Biology and Plant Biochemistry, Regensburg Center for Biochemistry, University of Regensburg, Universitätsstraße 31, 93053 Regensburg, Germany

2 Laboratory of Biochemistry, Wageningen University, Stippeneng 4, 6708WE Wageningen, The Netherlands
Method Papers can be submitted in a relatively free format including a protocol style that has a stronger emphasis on the detailed implementation of an established, recently developed method, but may also describe the development or adaptation of a method for plant reproduction research, as well as results and insights obtained with the method. Any combination of these different types is also welcome. For this special issue, we collected Method Papers with a focus on cellular omics methods involving the isolation of highly specific reproductive stages. Papers report the isolation of gametic cells and early embryos as well as endosperm for transcriptomic and proteomic studies in various plant species including Arabidopsis thaliana, Amborella trichopoda, tobacco and rice.

In a protocol style, Li et al. (2019) described step-by-step how gametes (egg and sperm cells) can be isolated from rice at sufficient quantities for omics studies. Viable egg cells are manually captured from dissected ovaries without fixation or enzymatic treatments. The same procedure can also be used to isolate zygotes at defined time points after pollination. Authors further described how rice sperm cells can be isolated at large and pure quantities using a blender method coupled with Percoll density gradient centrifugation. A general workflow is presented that includes details on how RNA contamination from surrounding cells can be determined.

Flores-Tornero et al. (2019) provided a protocol for the manual isolation of egg apparatus cells from the basal angiosperm Amborella trichopoda. This represents the first report about egg cells from this species, which are difficult to distinguish from synergid cells. Little is known about reproductive mechanisms in basal angiosperms, and molecular markers are not available. The authors subdivided isolated cells by size in three categories and determined expression profiles by RNA-seq. Genes enriched in the larger cells containing homologs of the Arabidopsis egg cell-specific gene family ECl (Sprunck et al. 2012) and enrichment of genes encoding various CRPs [secreted cysteine-rich peptides including a clade IV RALF; (Murphy and De Smet 2014)] in the smaller cells suggest that egg cells are larger than synergid cells. The procedure described is likely also applicable to 
other plant species and provides a guideline for the isolation of egg apparatus cells in general. The authors further suggested that single cells should be analyzed in future studies.

The idea of single-cell analysis is elaborated by Misra et al. (2019). The authors provided a very useful workflow diagram and a detailed protocol on how single-sperm cells can be isolated from bursting pollen tubes of A. thaliana. Using RNA-seq, the authors compared transcriptomes among single-sperm cells with those of bulks of sperm cells (50 cells). High variation in gene expression profiles was observed between single-sperm cells and pooled cells, showing that many expressed genes are not detected in studies at the single-cell level. Considering the increasing demand of single-cell analyses, the authors are optimistic that technical optimization will diminish these limitations.

Cell fate acquisition is often associated with changes in chromatin structure and accessibility. For large-scale studies using various genotypes and tissues, simple and robust methods are required to study, for example, histone marks. Using the model plant A. thaliana, Borg et al. (2019) now describe a detailed 2-day protocol to isolate pollen nuclei and immunostain these for histone marks. Moreover, they report how epitopes can be de-masked through antigen retrieval, which significantly improves signal intensity of antibodies targeting heterochromatic histone marks.

A technique named Automated Stack Iterative Subtraction Tool (ASIST) was developed by Ponvert et al. (2018) for measuring pollen tube phenotypes. The method can be applied both to in vitro and in vivo grown pollen tubes. ASIST converts growing pollen tube tips into closed particles allowing to trace individual tubes within hundreds of pollen tubes. This allows to study pollen tube growth behaviors and thus facilitates pollen tube phenomics at large scale.

Following double fertilization, two products form: the embryo and the endosperm, each with unique developmental patterns and functions. Several papers present methods for expression and chromatin analysis in endosperm or embryo, or cell types thereof.

Del Toro-De Léon and Köhler (2019) described the use of a method for affinity-based isolation of nuclei from the developing endosperm to collect mRNA profiles. This method—named INTACT—uses a two-component labeling system to decorate the nuclear lamina with biotin that can be purified by magnetic beads (Deal and Henikoff 2010). Following their method, the authors showed that there is little contamination with other nuclei, and in addition, it is shown that the nuclear mRNA largely represents the total mRNA of endosperm. Using the data collected from inter-ecotype crosses in Arabidopsis, the authors identified a number of gene sets that are preferentially expressed from maternal or paternal genomes.

In contrast to mRNA expression, Zheng and Gehring (2019) probed histone methylation in endosperm chromatin.
The authors optimized the CUT\&RUN method (Skene and Henikoff 2017) that uses protection from a nuclease to identify the sites of protein binding in the genome. After FACSbased sorting of mitotic endosperm nuclei through their $6 \mathrm{n}$ ploidy, the authors used H3K27me3 antibodies, connected to the CUT\&RUN method, to define sites of this histone mark. Overall, the profile corresponded well to earlier INTACTbased profiles of the same mark in Arabidopsis endosperm (Moreno-Romero et al. 2016). Importantly, 25 times less nuclei were used in the present paper. Finally, the authors used the described method for inter-ecotype crosses to identify genes for which there is a parent-of-origin bias in histone methylation.

Four papers described methodologies to study gene expression in the Arabidopsis embryo or its cell types. Both Hofmann et al. (2019) and Gao et al. (2019) studied gene expression patterns in isolated Arabidopsis embryos throughout development and derived information on the successive waves of gene expression as the embryo develops. Hofmann et al. first optimized low mRNA-input sequencing procedures and generated high-quality datasets on a range of stages. Through data analysis, they showed that the Arabidopsis embryo has a unique transcriptome when compared to other stages of life, even differing substantially from somatic embryos. Using their data, they defined four distinct phases in Arabidopsis embryogenesis and identified gene expression markers unique to each phase. Gao et al. used their stage-specific transcriptomes to identify functions enriched in each stage, indicative of cellular and physiological processes at each stage of embryogenesis. Further, they identified stage-specific gene co-expression modules that may be involved in driving the cellular changes observed.

Two papers focused on the transcriptional difference between cells within the Arabidopsis embryo. Zhou et al. (2019) described a procedure for isolating mRNA from either the apical or basal cell in the young Arabidopsis embryo. The same team had previously used this approach in tobacco, a species with much larger embryos and cells (Ma et al. 2011). Through a combination of microdissection and laser ablation, the authors managed to obtain pure RNA without significant contamination from non-embryo cells. Undoubtedly, the RNA-seq data obtained will be very useful in studying differences between embryonic and nonembryonic cells.

Palovaara and Weijers (2019) described a method to isolate nuclei from different cell types in the developing Arabidopsis embryo following an INTACT-based procedure the same authors have published recently (Palovaara et al. 2017). The embryo is encapsulated in seed and fruit, and cell types in the embryo (e.g., vascular) are shared with surrounding tissues, making the isolation of embryoderived cells of such types very challenging. The authors harnessed the power of two-component INTACT labeling 
by expressing one component in a tissue-specific manner and the other in an embryo-specific manner. The authors described methodological details of the procedure and used the INTACT-derived data to compare nuclear to cellular mRNA in the early embryo. They found that while there is general concordance, there are interesting differences as well. For example, mRNA stability appears to contribute to nuclear/cytoplasmic differences, and several transcription factor transcripts seem selectively enriched or depleted in nuclear mRNA.

We believe that this selection of Method Papers will be very useful for the plant reproduction community and beyond. Most of the methods and protocols provided in the special issue are described and optimized for A. thaliana. These protocols will likely have to be adapted for other plant species, and/or alternative protocols may have to be established. Nonetheless, we anticipate that future Method Papers, both in regular and special issues of Plant Reproduction, will push the technological frontier in this exciting research field and we thus invite the research community to continue submitting Method Papers for future issues.

\section{References}

Borg M, Buendia D, Berger F (2019) A simple and robust protocol for immunostaining Arabidopsis pollen nuclei. Plant Reprod $32: 39-44$

Deal RB, Henikoff S (2010) A simple method for gene expression and chromatin profiling of individual cell types within a tissue. Dev Cell 18:1030-1040

Del Toro-De León G, Köhler C (2019) Endosperm-specific transcriptome analysis by applying the INTACT system. Plant Reprod 32:55-62

Flores-Tornero M, Proost S, Mutwil M, Scutt CP, Dresselhaus T, Sprunck S (2019) Transcriptomics of manually isolated Amborella trichopoda egg apparatus cells. Plant Reprod 32:15-28

Gao P, Xiang D, Quilichini TD, Venglat P, Pandey PK, Wang E, Gillmor CS, Datla R (2019) Gene expression atlas of embryo development in Arabidopsis. Plant Reprod 32:93-104
Hofmann F, Schon MA, Nodine MD (2019) The embryonic transcriptome of Arabidopsis thaliana. Plant Reprod 32:77-92

Li C, Xu H, Russell SD, Sundaresan V (2019) Step-by-step protocols for rice gamete isolation. Plant Reprod 32:5-14

Ma L, Xin H, Qu L, Zhao J, Yang L, Zhao P, Sun MX (2011) Transcription profile analysis reveals that zygotic division results in uneven distribution of specific transcripts in apical/basal cells of tobacco. PLoS ONE 6:e15971

Misra CS, Santos MR, Rafael-Fernandes M, Martins NP, Monteiro M, Becker JD (2019) Transcriptomics of Arabidopsis sperm cells at single-cell resolution. Plant Reprod 32:29-38

Moreno-Romero J, Jiang H, Santos-Gonzales J, Köhler C (2016) Parental epigenetic asymmetry of PRC2-mediated histone modifications in the Arabidopsis endosperm. EMBO J 35:1298-1311

Murphy E, De Smet I (2014) Understanding the RALF family: a tale of many species. Trends Plant Sci 19:664-671

Palovaara J, Weijers D (2019) Adapting INTACT to analyse cell-typespecific transcriptomes and nucleocytoplasmic mRNA dynamics in the Arabidopsis embryo. Plant Reprod 32:113-122

Palovaara J, Saiga S, Wendrich JR, van 't Wout Hofland N, van Schayck JP, Hater F, Mutte S, Sjollema J, Boekschoten M, Hooiveld GJ, Weijers D (2017) Transcriptome dynamics revealed by a gene expression atlas of the early Arabidopsis embryo. Nat Plants 3:894-904

Ponvert N, Goldberg J, Leydon A, Johnson MA (2018) Iterative subtraction facilitates automated, quantitative analysis of multiple pollen tube growth features. Plant Reprod 32:45-54

Skene PJ, Henikoff S (2017) An efficient targeted nuclease strategy for high-resolution mapping of DNA binding sites. eLife 6:e21856

Sprunck S, Rademacher S, Vogler F, Gheyselinck J, Grossniklaus U, Dresselhaus T (2012) Egg cell-secreted EC1 triggers sperm cell activation during double fertilization. Science 338:1093-1097

Zheng XY, Gehring M (2019) Low-input chromatin profiling in Arabidopsis endosperm using CUT\&RUN. Plant Reprod 32:63-76

Zhou X, Shi C, Zhao P, Sun MX (2019) Isolation of living apical and basal cell lineages of early proembryos for transcriptome analysis. Plant Reprod 32:105-112

Publisher's Note Springer Nature remains neutral with regard to jurisdictional claims in published maps and institutional affiliations. 